\title{
Outcomes after the conversion of metal-on-metal hip resurfacing arthroplasty to ceramic-on-ceramic total hip arthroplasty: A retrospective observational study with a mean 10-year follow-up
}

\section{Weiguang Yu}

Sun Yat-sen University First Affiliated Hospital

\section{Mao Shuai}

Sun Yat-sen University First Affiliated Hospital

Jinluan Lin

The Affiliated hospital of Fujian Medical University

Baomin Chen

Sun Yat-sen University First Affiliated Hospital

Mingdong Zhao

Jinshan Hospital, Fudan University

Chao Xin Zhang ( $\square$ zhangxc401@aliyun.com ) Jinshan Hospital, Fudan University

Wenli Chen

Sun Yat-sen University First Affiliated Hospital

Guowei Han

Sun Yat-sen University First Affiliated Hospital

Research article

Keywords:

Posted Date: January 21st, 2020

DOI: https://doi.org/10.21203/rs.2.21421/v1

License: (c) (i) This work is licensed under a Creative Commons Attribution 4.0 International License. Read Full License 


\section{Abstract}

\section{Background}

The purpose of this retrospective study was to assess clinical outcomes following failed metal-on-metal hip resurfacing arthroplasty (MoM-HRA) revised using ceramic-on-ceramic total hip arthroplasty (CoCTHA) via the direct anterior approach (DAA).

Methods

Data involving 112 patients (112 hips) with failed primary MoM-HRA that was revised using CoC-THA via the DAA during 2006 - 2018 were retrospectively analysed. The mean age was 54.6 years (45-63 years). Frequent surgical indications for conversion were aseptic loosening and femoral neck fracture. The primary endpoint was the Postel-Merle d'Aubigne functional score (PMA). Secondary endpoints were the major orthopaedic complication rate and serum metal ion levels (chromium and cobalt).

\section{Results}

The mean follow-up time was 10 years (range, 4 - 13 years). The mean PMA improved from 9 (4-14) to 16 $(13-18)(p=0.001)$. Six patients had undergone a re-revision intervention. Fourteen incidents of aseptic loosening and four periprosthetic fractures were observed. A consistent decline in mean serum metal ion levels was detected [chromium decreased from $36.6 \mu \mathrm{g} / \mathrm{L}(12.5-76.5 \mu \mathrm{g} / \mathrm{L})$ prior to conversion to $2.6 \mu \mathrm{g} / \mathrm{L}$ $(0.1-13.5 \mu \mathrm{g} / \mathrm{L})$ at final follow-up $(p=0.001)$, cobalt decreased from $37.5 \mu \mathrm{g} / \mathrm{L}(6.7-93.2 \mu \mathrm{g} / \mathrm{L})$ to $2.2 \mu \mathrm{g} / \mathrm{L}$ $(1.2-18.4 \mu \mathrm{g} / \mathrm{L})(\mathrm{p}=0.003)]$.

Conclusion

Failed primary MoM-HRA converted to CoC-THA using the DAA yielded acceptable clinical outcomes.

\section{Background}

Failure of standard total hip arthroplasty (THA) triggered by an adverse reaction to metal debris (ARMD)induced osteolysis can be prevented by metal-on-metal hip resurfacing arthroplasty (MoM-HRA)[1-3]. However, the use of MoM-HRA has frequently been conditioned by bone conditions[4, 5]. Recurrent shortterm failures are well recognized for MoM-HRA, particularly MoM bearings in individuals with small femoral components, and they are commonly addressed with a bipolar revision with a conversion to ceramic-on-ceramic THA (CoC-THA) [6, 7]. Failure secondary to MoM wear has become a concern[2, 8]. ARMD-induced osteolysis is overwhelmingly implicated in the pathophysiology of MoM-HRA failure, contributing to as high as a 10 to $27 \%$ revision rate and quick time-to-failure[9-11].

Limited studies have assessed complications from the conversion of MoM-HRA to CoC-THA. Interest in CoC-THA has increased over the last decade[6, 7]. Numerous reports have demonstrated that the outcomes of conversion CoC-THA are mostly influenced by the reason for conversion[7, 2, 12]. 
Additionally, there remain concerns regarding whether MoM-HRA conversion to CoC-THA is associated with poor clinical outcomes[6, 9].

To date, consensus remains lacking regarding long-term outcomes after MoM-HRA conversion to CoCTHA. To gain a comprehensive understanding of the long-term results of these conversions, we performed this retrospective study to evaluate the long-term clinical outcomes of failed primary MoMHRA conversion to CoC-THA using the direct anterior approach (DAA).

\section{Methods}

\section{Study population}

Between February 2006 and December 2018, an original study cohort of 186 patients (212 hips) who underwent conversion of a failed primary MoM-HRA to a CoC-THA was obtained from our joint surgery practice database (Sun Yat-sun University, Guangzhou, China), which was established in 2002 and contains data derived from computerized records. A detailed description of the database is available in our previous report[13]. The inclusion criteria were as follows: patients who underwent conversion of an original MoM-HRA (uncemented cup: Dynacup, Tornier, Montbonnot, France; a PE insert: Selene, Tornier) to a CoC-THA (ceramic-on-ceramic, Stryker, Mahwah, New Jersey); and patients who had no exposure to other known sources of chromium or cobalt or impaired renal function. The main exclusion criteria were deficient clinical outcome data, dyskinesia prior to conversion, joint-related diseases, lack of ability to follow instructions, active systemic or local infection, cancer, an injury-severity score (ISS) of more than 10 , serious organ complications (i.e., brain, pulmonary, cardiovascular, renal), cognitive impairment or psychosis, drug abuse, body mass index $(\mathrm{BMI})>40 \mathrm{~kg} / \mathrm{m}^{2}$, and an American Society of Anesthesiologists (ASA) score of IV or V.

The revision surgery was performed by the 3 high-volume surgeons (WY, XZ, MZ) using the DAA in the supine position on a traction table, as previously described[6]. The indications for conversion involved aseptic loosening, femoral neck fracture, instability, and intolerable hip pain. A consistent protocol of antibiotic application was performed perioperatively for each patient. An anticoagulant regimen was applied to all patients without contraindications for one month after conversion. The same rehabilitation program was used for all patients. Full weight-bearing was allowed from the day after conversion.

The primary outcome was the Postel-Merle d'Aubigne functional score (PMA). Secondary outcomes were the rates of major orthopaedic complications (re-revision, aseptic loosening, periprosthetic fracture, dislocation, deep infection, intolerable hip pain) and serum metal ion levels (chromium and cobalt). Data were collected at the baseline evaluation and the final clinical review by WY and XZ. All patients who underwent CoC-THA were evaluated individually using the PMA at each follow-up. Measurements of the PMA occurred 1 year, 2 years, and every 2 years after conversion. Image data obtained at these same time points consisted of an anteroposterior (AP) pelvis view and AP and lateral views of the hip and were assessed by experienced radiologists. The abduction angle of the cup, measured as reported previously, 
was assessed on the pelvic side. Analyses of blood trace elements were performed at the Central Laboratory of Physics and Chemistry, Sun Yat-sen University (Guangzhou, China). Measurements of serum metal ion levels occurred 1 year, 2 years, and every 1 year after conversion. The procedures for the measurements are based on previous descriptions $[6,14-17]$ and our laboratory trace element detection guide.

To ensure the validity of these data, all study data were audited by the two co-authors (WY and GH). Based on the inclusion and exclusion criteria in this study, a total of 112 eligible patients (112 hips) undergoing conversion of a primary MoM-HRA to a CoC-THA were included in the final analysis. The mean age was 54.6 years (45-63 years). The mean interval to failure after initial MoM-HRA revision was 3.4 years (22 days -5.6 years). At the time of analysis, the mean follow-up after MoM-HRA revision was 10.1 years (4.5-13.6 years). A study flow chart is shown in Figure 1, and the patient characteristics are described in Table 1.

\section{Statistical analysis}

Follow-up was defined as the interval from the date of MoM-HRA revision to the date of death or the last follow-up, whichever came first. Aseptic loosening was defined as in previous reports[18, 19]. Re-revision was defined as the removal or exchange of any component. Osteolysis was defined as expanding areas of bone resorption surrounding the prosthesis (cup and/or stem). Continuous variables are presented as the means with standard deviations (SD) and ranges and were analysed using Student's t tests.

Categorical data are presented as frequencies and percentages and were analysed by Pearson's $\chi^{2}$ test or Fisher's exact test, as appropriate. Statistical analyses were performed using SPSS, version 24.0 (IBM, Armonk, NY). A 2-sided $p$ value $<0.05$ was considered significant.

\section{Results}

The mean PMA after revision is presented in Table 2. CoC-THA yielded acceptable functional outcomes. The PMA improved from $9(4-14)$ prior to revision to $16(13-18)$ at the final follow-up $(p=0.000)$. Approximately $86 \%$ of the patients with a failed MoM-HRA that was converted using a CoC-THA had a satisfactory PMA at the final follow-up.

Prior to the 3 years after revision, no fracture around the prosthesis or dysesthesia on the affected limb was reported for any patient. Thirty-one major orthopaedic complications were detected in the cohort (Table 3). Of these complications, 6 (5.3\%) required re-revision surgery, 14 (12.5\%) suffered aseptic loosening, 4 (3.5\%) suffered periprosthetic fracture, 3 (2.6\%) suffered dislocation, 2 (1.7\%) suffered deep infection, and $2(1.7 \%)$ suffered intolerable hip pain. Nearly $82.1 \%$ of re-revisions were attributed to aseptic loosening. The mean concentration of serum chromium decreased from $36.6 \mu \mathrm{g} / \mathrm{L}(12.5-76.5$ $\mu \mathrm{g} / \mathrm{L})$ prior to revision to $2.6 \mu \mathrm{g} / \mathrm{L}(0.1-13.5 \mu \mathrm{g} / \mathrm{L})$ at the final follow-up $(p=0.001)$, and the mean concentration of serum cobalt decreased from $35.8 \mu \mathrm{g} / \mathrm{L}(6.3-85.5 \mu \mathrm{g} / \mathrm{L})$ to $2.2 \mu \mathrm{g} / \mathrm{L}(1.2-18.4 \mu \mathrm{g} / \mathrm{L})(p=$ 0.001). Three years after revision, a significant reduction was noted in the mean concentration of serum 
chromium (Table 4). At the final follow-up, 27 patients had serum chromium levels less than $1 \mu \mathrm{g} / \mathrm{L}$, and 32 had serum cobalt levels less than $1 \mu \mathrm{g} / \mathrm{L}$.

\section{Discussion}

This retrospective analysis characterizing the long-term outcomes of a failed primary MoM-HRA that was converted with a CoC-THA provides evidence that the conversion of a failed MoM-HRA to a CoC-THA is acceptable, with serum metal ion levels markedly decreasing. To our knowledge, this is a relatively large sample study regarding conversion CoC-THA outcomes following a failed MoM-HRA.

Several limitations should be acknowledged in this study. First, the retrospective nature of this study is vulnerable to errors in assessing baseline data and can result in attenuated power to draw strong conclusions. An attempt was made to avoid confounding variables; however, the resulting analysis may be deficient. Second, selection bias is almost impossible to avoid owing to the elimination of unexpected patients. Third, potential variables related to the expertise of the surgeons may have affected the results. However, complying with all recommendations and recognized guidelines and having a high volume of practice enabled us to optimize patient care and decrease the risk of complications. Despite these limitations, we reckoned that the error margin appeared to be allowable in this setting.

Limited prospective multi-centre studies have assessed outcomes of conversion CoC-THA for a failed MoM-HRA[20, 21]. Findings from published retrospective studies have revealed functional advantages with CoC-THA[9, 22]. However, there remain concerns about the suitability of CoC-THA[23]. Several studies have verified that conversion CoC-THA for failed MoM-HRA is associated with acceptable functional outcomes[12, 20]. However, an increasing but still exceedingly limited body of literature has assessed the role of conversion CoC-THA following a failed primary MoM-HRA and demonstrated that the functional outcomes for CoC-THA were susceptible to patient indications[8].

A low re-revision rate was documented after conversion CoC-THA following a failed MoM-HRA. Matharu et al. [24] noticed that $6 \%$ of cases required re-revision in a series of 48 failed MoM-HRAs converted to CoC-THAs. This may partly be attributed to the DAA because it potentially improves dynamic hip stability and has the ability to decrease the risk of dislocation after conversion[6]. Additionally, a reduced rate of complications was observed in a study by Migaud et al.[25] that assessed CoC-THA via the DAA. Notably, no dislocation was observed in their cohort at a mid-term follow-up. Combined with the current findings, the results confirmed by these studies appear to verify that the DAA is the preferred surgical approach for converting a failed MoM-HRA. Metal ions can decrease the number of osteoblast cells by inhibiting their gene expression, theoretically increasing the risk of MoM-HRA failure and creating unnecessary obstacles for a future conversion[26, 27]. The present results for failed MoM-HRA conversion to CoC-THA have also shown low rates of re-revision or aseptic loosening. In Europe [28], the five-year and ten-year revision rates for MoM-HRA and stemmed large head MoM implants were higher than for CoC-THA. Hip stability following cemented CoC-THA is superior to that reported for uncemented CoC-THA because of the enhanced stability of bone and cemented components. The mechanical wear of the acetabular 
component tends to be related to uncommonly high wear initiated by encumbered edge loading[4, 29]. A prospective study[20] of 39 hips with between 1 and 4 years of follow-up exhibited a low rate of rerevision for CoC-THA following a failed MoM-HRA, predominantly owing to the high stability of the acetabular component after conversion CoC-THA.

The risk associated with conversion of MoM-HRA to CoC-THA is still an extensive concern[30, 8]. Nevertheless, the available studies on outcomes of the conversion remain deficient[31]. Savarino et al.[26] reported the role of CoC-THA and revealed noteworthy dissimilarities regarding conclusions about it. Attention has been paid to whether CoC-THA has a low rate of either re-revision or aseptic loosening according to long-term outcomes[32]. Bouveau et al. [6] assessed 13 conversions of MoM-HRA to CoCTHA and showed a low re-revision rate (6\%), primarily due to the aseptic loosening of acetabular components. Using an analogous premise, Stryker et al.[33] reported 65 conversions of MoM-HRA to CoCTHA and demonstrated a low re-revision rate (7\%), mainly ascribed to aseptic loosening $(76 \%)$ and deep infection (16\%). Recently, Rahman et al. [8] reported on 25 conversions and showed that none of the patients suffered aseptic loosening. In brief, documented results of MoM-HRA revised using CoC-THA have exhibited an exceedingly low rate of re-revision, and this conversion tends to be a safe choice for decreasing the re-revision rate.

Earlier studies have shown that the serum metal ion levels, mainly for chromium ions, remain at moderate to high levels after conversion CoC-THA in the elderly population[34, 35]. The return to exceptionally low or unnoticeable levels could take more than 3 years[36], which is in accordance with our follow-up outcomes that serum chromium ion levels remain moderately high in a majority of patients 3 years after conversion. Urinary excretion of chromium has a significant effect on decreasing serum chromium ion levels[35]. Regrettably, several reports have shown that the amount of chromium ions excreted in urine is extremely small and that the accumulation of chromium ions predominantly occurs in tissues and erythrocytes[26, 16]. Consequently, significant reductions in serum chromium ion levels will be extremely challenging to achieve following an increase[16]. Amstutz et al.[36] assessed 18 patients who were treated with MoM-HRA in a prospective study and concluded that serum chromium ions escalated within the 1st year and then declined and stabilized. Additionally, they pointed out that serum chromium ions in a well-functioning endoprosthesis appear to be low and fail to increase with time. Furthermore, a sharp increase in serum metal ions has been shown to be related to prosthesis failure[37]. Several studies[20, 36,38 report the toxic effects of prolonged elevated systemic metal ion exposure; therefore, whole blood metal ion testing should have a place in follow-up. Persistent low-dose exposure to chromium ions in patients with conversion of a failed MoM-HRA to a CoC-THA may have systemic effects and to date, longterm epidemiological studies do not seem to have been performed[36].

\section{Conclusions}

The long-term results reported in this study support an increasing body of evidence that conversion CoCTHA for a failed primary MoM-HRA was associated with a noteworthy improvement in clinical outcome, along with PMAs, re-revision rates, and rates of aseptic loosening, as well as significantly reduced serum 
metal ion levels. However, based on the data presented here, to determine other clinical benefits to, for example, the orthopaedic complication rate and prosthesis survival curve, future prospective multi-centre studies are needed.

\section{Abbreviations}

MoM-HRA: metal-on-metal hip resurfacing arthroplasty; CoC-THA: ceramic-on-ceramic total hip arthroplasty; DAA: direct anterior approach; ARMD: adverse reaction to metal debris; BMD: bone mineral density; ISS: injury severity score; BMI: body mass index; ASA: American Society of Anesthesiologists; PMA: Postel-Merle d'Aubigne; AP: anteroposterior; SD: Standard deviation.

\section{Declarations}

\section{Acknowledgements}

The authors would like to thank Lu Yu for her help with retrieval of data.

\section{Funding}

Funding for this research was received from the National Natural Science Foundation of China (Grant No. 81971315).

\section{Availability of data and materials}

The datasets used and/or analysed during the current study are available from the corresponding author upon reasonable request.

\section{Authors' contributions}

WY and SM performed the data collection and analysis and participated in manuscript writing. JL and BC performed the database setup and statistical analysis. MZ, XZ, WY, and GH performed the operations. WC and $X Z$ participated in the study design and coordination and helped to draft the manuscript. All authors have read and approved the final manuscript.

\section{Ethics approval and consent to participate}

This study was approved by the Medical Ethics Committee (The First Affiliated Hospital, Sun Yat-sen University), and an exemption from informed consent was obtained from our responsible Investigational Ethics Review Board.

\section{Consent for publication}

Not applicable. 


\section{Competing interests}

The authors declare that they have no competing interests.

\section{References}

1. Lee R, Essner A, Wang AG. Tribological considerations in primary and revision metal-on-metal arthroplasty. J Bone Joint Surg Am. 2008;90A:118-24.

2. De Haan R, Campbell PA, Su EP et al. Revision of metal-on-metal resurfacing arthroplasty of the hip The influence of malpositioning of the components. J Bone Joint Surg Br. 2008;90B(9):1158-63.

3. Scholes CJ, Ebrahimi M, Farah SB et al. The outcome and survival of metal-on-metal hip resurfacing in patients aged less than 50 years A PROSPECTIVE OBSERVATIONAL COHORT STUDY WITH MINIMUM TEN-YEAR FOLLOW-UP. Bone Joint J. 2019;101B(1):113-20.

4. Barrett WP, Kindsfater KA, Lesko JP. Large-Diameter Modular Metal-on-Metal Total Hip Arthroplasty Incidence of Revision for Adverse Reaction to Metallic Debris. J Arthroplasty. 2012;27(6):976-83.

5. Borton ZM, Mumith AS, Nicholls AJ et al. The Outcome of Revision Surgery for Failed Metal-on-Metal Total Hip Arthroplasty. J Arthroplasty. 2019;34(8):1749-54.

6. Bouveau $\mathrm{V}$, Haen TX, Poupon $\mathrm{J}$ et al. Outcomes after revision of metal on metal hip resurfacing to total arthroplasty using the direct anterior approach. Int Orthop. 2018;42(11):2543-8.

7. Crawford DA, Adams JB, Morris MJ et al. Revision of Failed Metal-on-Metal Total Hip Arthroplasty: Midterm Outcomes of 203 Consecutive Cases. J Arthroplasty. 2019;34(8):1755-60.

8. Rahman WA, Amenabar T, Hetaimish BM et al. Outcome of Revision Total Hip Arthroplasty in Management of Failed Metal-on-Metal Hip Arthroplasty. J Arthroplasty. 2016;31(11):2559-63.

9. Gross TP, Liu F. Outcomes After Revision of Metal-on-Metal Hip Resurfacing Arthroplasty. J Arthroplasty. 2014;29(9):219-23.

10. Iqbal HJ, Al-Azzani WAK, Jackson-Taylor E et al. Outcome of revision arthroplasty for failed metal-onmetal total hip replacements; is there a relation with metal ions? Hip Int. 2017;27(3):235-40.

11. Jennings JM, White S, Martin JR et al. Revisions of Modular Metal-on-metal THA Have a High Risk of Early Complications. Clin Orthop Relat Res. 2019;477(2):344-50.

12. Lainiala OS, Reito AP, Nieminen JJ et al. Declining Revision Burden of Metal-on-Metal Hip Arthroplasties. J Arthroplasty. 2019;34(9):2058-+.

13. Yu W, Zhang $X$, Zhu $X$ et al. Proximal femoral nails anti-rotation versus dynamic hip screws for treatment of stable intertrochanteric femur fractures: an outcome analyses with a minimum 4 years of follow-up. BMC Musculoskelet Disord. 2016;17:222.

14. Ahmed SS, Bawale R, Jain S et al. Metal ion levels comparison: Metal-on-metal hip resurfacing vs total hip arthroplasty in patients requiring revision surgery. J Orthop. 2018;15(4):1013-6.

15. Carlson BC, Bryan AJ, Carrillo-Villamizar NT et al. The Utility of Metal lon Trends in Predicting Revision in Metal-on-Metal Total Hip Arthroplasty. J Arthroplasty. 2017;32(9):S214-S9. 
16. Robinson PG, Wilkinson AJ, Meek RMD. Metal ion levels and revision rates in metal-on-metal hip resurfacing arthroplasty: a comparative study. Hip Int. 2014;24(2):123-8.

17. Grammatopoulos G, Munemoto M, Pollalis A et al. Correlation of serum metal ion levels with pathological changes of ARMD in failed metal-on-metal-hip-resurfacing arthroplasties. Arch Orthop Trauma Surg. 2017;137(8):1129-37.

18. Watts $C D$, Abdel MP, Hanssen AD et al. Anatomic Hip Center Decreases Aseptic Loosening Rates After Total Hip Arthroplasty with Cement in Patients with Crowe Type-II Dysplasia A Concise Followup Report at a Mean of Thirty-six Years. J Bone Joint Surg Am. 2016;98(11):910-5.

19. Petis SM, Kubista B, Hartzler RU et al. Polyethylene Liner and Femoral Head Exchange in Total Hip Arthroplasty Factors Associated with Long-Term Success and Failure. J Bone Joint Surg Am. 2019;101(5):421-8.

20. Liddle AD, Satchithananda K, Henckel J et al. Revision of metal-on-metal hip arthroplasty in a tertiary center A prospective study of 39 hips with between 1 and 4 years of follow-up. Acta Orthop. 2013;84(3):237-45.

21. Schouten R, Malone AA, Tiffen $\mathrm{C}$ et al. A prospective, randomised controlled trial comparing ceramicon-metal and metal-on-metal bearing surfaces in total hip replacement. J Bone Joint Surg Br. 2012;94B(11):1462-7.

22. Konan S, Garbuz DS, Masri BA et al. The Outcomes Following Revision of Monoblock Metal on Metal Acetabular Components for Painful Micromotion in the Absence of Adverse Local Tissue Reaction to Metal. J Arthroplasty. 2017;32(3):915-8.

23. Matharu GS, Judge A, Murray DW et al. Outcomes After Metal-on-metal Hip Revision Surgery Depend on the Reason for Failure: A Propensity Score-matched Study. Clin Orthop Relat Res. 2018;476(2):245-58.

24. Matharu GS, Pandit HG, Murray DW. Poor Survivorship and Frequent Complications at a Median of 10 Years After Metal-on-Metal Hip Resurfacing Revision. Clin Orthop Relat Res. 2017;475(2):304-14.

25. Migaud H, Putman S, Kern G et al. Do the Reasons for Ceramic-on-ceramic Revisions Differ From Other Bearings in Total Hip Arthroplasty? Clin Orthop Relat Res. 2016;474(10):2190-9.

26. Savarino L, Padovanni G, Ferretti M et al. Serum Ion Levels after Ceramic-on-Ceramic and Metal-onMetal Total Hip Arthroplasty: 8-Year Minimum Follow-up. J Orthop Res. 2008;26(12):1569-76.

27. Seppanen M, Laaksonen I, Pulkkinen P et al. High Revision Rate for Large-head Metal-on-metal THA at a Mean of 7.1 Years: A Registry Study. Clin Orthop Relat Res. 2018;476(6):1223-30.

28. Pijls BG, Meessen J, Tucker $\mathrm{K}$ et al. MoM total hip replacements in Europe: a NORE report. Efort Open Reviews. 2019;4(6):423-9.

29. Bjorgul K, Novicoff WN, Andersen ST et al. High rate of revision and a high incidence of radiolucent lines around Metasul metal-on-metal total hip replacements RESULTS FROM A RANDOMISED CONTROLLED TRIAL OF THREE BEARINGS AFTER SEVEN YEARS. Bone Joint J. 2013;95B(7):881-6.

30. Porat M, Parvizi J, Sharkey PF et al. Causes of Failure of Ceramic-on-Ceramic and Metal-on-Metal Hip Arthroplasties. Clin Orthop Relat Res. 2012;470(2):382-7. 
31. Lainiala O, Reito A, Elo P et al. Revision of Metal-on-metal Hip Prostheses Results in Marked Reduction of Blood Cobalt and Chromium lon Concentrations. Clin Orthop Relat Res. 2015;473(7):2305-13.

32. Pattyn C, De Smet KA. Primary Ceramic-on-Ceramic Total Hip Replacement Versus Metal-on-Metal Hip Resurfacing in Young Active Patients. Orthopedics. 2008;31(11):1078-82.

33. Stryker LS, Odum SM, Fehring TK et al. Revisions of Monoblock Metal-on-metal THAs Have High Early Complication Rates. Clin Orthop Relat Res. 2015;473(2):469-74.

34. Sangaletti R, Spreafico A, Barbieri F et al. Metal ion trend in patients with metal-on-metal total hip arthroplasty: a 10-year prospective study. Hip Int. 2018;28:43-7.

35. Engh CA, MacDonald SJ, Sritulanondha S et al. Metal Ion Levels After Metal-on-Metal Total Hip Arthroplasty A Five-Year, Prospective Randomized Trial. J Bone Joint Surg Am. 2014;96A(6):448-55.

36. Amstutz HC, Campbell PA, Dorey FJ et al. Do lon Concentrations after Metal-on-Metal Hip Resurfacing Increase Over Time? A Prospective Study. J Arthroplasty. 2013;28(4):695-700.

37. Lee YK, Yoon BH, Choi YS et al. Metal on Metal or Ceramic on Ceramic for Cementless Total Hip Arthroplasty: A Meta-Analysis. J Arthroplasty. 2016;31(11):2637-45.

38. Bosker BH, Ettema HB, van Rossum $M$ et al. Pseudotumor formation and serum ions after large head metal-on-metal stemmed total hip replacement. Risk factors, time course and revisions in 706 hips. Arch Orthop Trauma Surg. 2015;135(3):417-25.

\section{Tables}

Table 1 Patient demographics at the time of MoM-HRA revision 


\begin{tabular}{|c|c|}
\hline Variable & CoC-THA $(n=112)$ \\
\hline Sex, M/F & $52 / 60$ \\
\hline Age, years & $54.6 \pm 9.2$ \\
\hline BMI, $\mathrm{kg} / \mathrm{m}^{2}$ & $27.8 \pm 6.6$ \\
\hline BMD & $-3.5 \pm 0.4$ \\
\hline Side, left/right & $54 / 58$ \\
\hline Interval to failure after primary MoM-THA & $3.4(22$ days -5.6 yrs $)$ \\
\hline \multicolumn{2}{|l|}{ Comorbidities, $\mathrm{n} \%$} \\
\hline Hypertension & $43(38.3)$ \\
\hline Diabetes mellitus & $27(24.1)$ \\
\hline Hypertension and diabetes mellitus & 15(13.3) \\
\hline \multicolumn{2}{|l|}{ Failure mode, n\% } \\
\hline Aseptic loosening & $69(61.6)$ \\
\hline Femoral neck fracture & $21(18.7)$ \\
\hline Pain & $16(14.2)$ \\
\hline Instability & $6(5.3)$ \\
\hline \multicolumn{2}{|l|}{ Original diagnosis, $\mathrm{n} \%$} \\
\hline Primary arthritis & $28(25.0)$ \\
\hline Secondary arthritis & $46(41.0)$ \\
\hline AVN & $44(39.2)$ \\
\hline \multicolumn{2}{|l|}{ ASA Index, n\% } \\
\hline [ & 16(20.3) \\
\hline Q & $78(69.6)$ \\
\hline & $18(16.0)$ \\
\hline \multicolumn{2}{|c|}{ Pre-revision serum metal ion concentrations, mean (range), $\mu \mathrm{g} / \mathrm{L}$} \\
\hline Chromium & $36.6(12.5-76.5)$ \\
\hline Cobalt & $37.5(6.7-93.2)$ \\
\hline Preoperative PMA & $9(4-14)$ \\
\hline Follow-up period (yrs) & $10.1(4.5-13.6)$ \\
\hline \multicolumn{2}{|c|}{$\begin{array}{l}\text { MoM-HRA: metal-on-metal hip resurfacing arthroplasty; CoC-THA: ceramic-on-ceramic } \\
\text { total hip arthroplasty; BMI: body mass index; BMD: bone mineral density; AVN: avascular } \\
\text { necrosis; ASA: American Society of Anesthesiologists; yrs: years; PMA: Postel-Merle } \\
\text { d'Aubigne. }\end{array}$} \\
\hline
\end{tabular}

\section{Table 2 Functional outcomes of conversion CoC-THA at each follow-up}

\begin{tabular}{lc}
\hline Time, year(s) after conversion & PMA \\
\hline 1 & $14(12-18)$ \\
2 & $15(13-18)$ \\
4 & $16(14-18)$ \\
6 & $16(14-18)$ \\
8 & $16(13-18)$ \\
10 & $16(13-18)$ \\
\hline Final follow-up & $16(13-18)$ \\
\hline CoC-THA: Ceramic-on-Ceramic total hip arthroplasty; PMA: Postel-Merle d'Aubigne.
\end{tabular}

\section{Table 3 Long-term outcomes of CoC-THA-related complications}




\begin{tabular}{ll}
\hline Variable, $\mathrm{n} \%$ & CoC-THA $(\mathrm{n}=112)$ \\
\hline Re-revision & $6(5.3)$ \\
\hline Aseptic loosening & $14(12.5)$ \\
\hline Periprosthetic fracture & $4(3.5)$ \\
Dislocation & $3(2.6)$ \\
\hline Deep infection & $2(1.7)$ \\
\hline Intolerable hip pain & $2(1.7)$ \\
\hline
\end{tabular}

Table 4 Post-revision serum metal ion at each follow-up

\begin{tabular}{lll}
\hline Time, year(s) after conversion & Chromium, $\mu \mathrm{g} / \mathrm{L}$ & Cobalt, $\mu \mathrm{g} / \mathrm{L}$ \\
\hline 1 & $12.2(2.6-57.9)$ & $9.7(7.4-72.3)$ \\
2 & $11.4(2.1-54.1)^{\#}$ & $8.8(6.2-56.4)$ \\
3 & $5.6(1.2-42.1)^{\#}$ & $7.6(6.1-42.8)$ \\
\hline 4 & $5.2(0.5-37.8)$ & $6.4(5.3-35.6)$ \\
\hline 5 & $4.3(0.4-32.6)$ & $6.1(4.4-31.2)$ \\
\hline 7 & $4.3(0.4-26.3)$ & $5.3(3.3-28.1)$ \\
\hline 8 & $3.6(0.3-21.1)$ & $4.5(3.1-26.3)$ \\
\hline 10 & $3.2(0.2-17.7)$ & $3.7(2.8-26.2)$ \\
Final follow-up & $2.9(0.1-14.2)$ & $3.1(2.5-24.5)$ \\
\hline
\end{tabular}

\# Significant difference. CoC-THA: ceramic-on-ceramic total hip arthroplasty; PMA: Postel-Merle d'Aubigne.

\section{Figures}


From February 2006 to December 2018, 186 patients (212 hips) who were treated with conversion of a failed primary MoM-HRA to CoC-THA was obtained from joint surgery practice database (Sun Yat-sun University, Guangzhou, China)

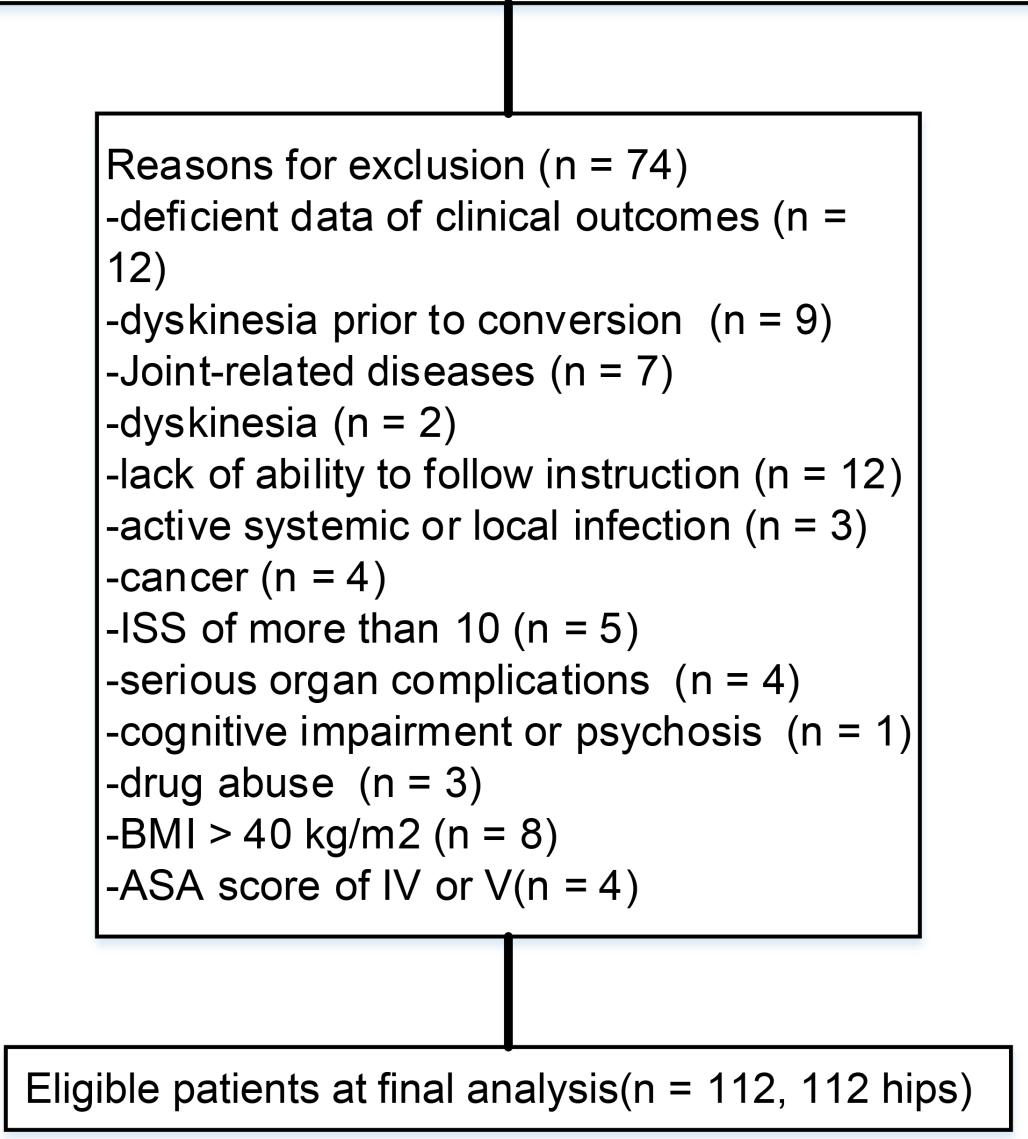

\section{Figure 1}

Flow diagram demonstrating methods for identification and exclusion of studies to assess clinical outcome following failed metal-on-metal hip resurfacing arthroplasty (MoM-HRA) revised using ceramicon-ceramic total hip arthroplasty (CoC-THA) per the direct anterior approach (DAA). 